\title{
LOGCONCAVITY OF THE COOLING OF A CONVEX BODY
}

\author{
GILLES DESLAURIERS AND SERGE DUBUC
}

\begin{abstract}
The solution $u(x, t)$ of the heat equation is logconcave in the space variable $x$ whenever the initial temperature $u_{0}(x)$ of the convex body is logconcave.
\end{abstract}

1. Introduction. A real function $f$ defined over a convex set is called logconcave if $f$ is a nonnegative function on $\Omega$ and if the following inequalities hold:

$$
f((1-t) x+t y) \geqslant(f(x))^{1-t}(f(y))^{t}
$$

when $x$ and $y$ belong to $\Omega$ and when $t$ is between 0 and 1. Let us consider a solution to the heat equation: $u(x, t)$ is defined for $x$ belonging to a bounded convex open subset of $\mathbf{R}^{d}$ and for $t>0$ and we assume that

$$
\frac{\partial u}{\partial t}=\frac{1}{2} \Delta u
$$

The following boundary conditions will be used:

(a) For every boundary point $a$ of $\Omega$ and every $t>0, \lim _{x \rightarrow a} u(x, t)=0$.

(b) For every point $x$ of $\Omega, \lim _{t \downarrow 0} u(x, t)=u_{0}(x)$ where $u_{0}(x)$ is a given function defined on $\Omega$.

The main result we will show is that the function $u(x, t)$ is logconcave in the space variable $x$ whenever the function $u_{0}(x)$ is logconcave. Otherwise said, if the initial temperature of a convex body is logconcave, at any time later the temperature of the body will still be logconcave in the space variable. From this fact, we will derive a property of Helmholtz's equation. If $\Omega$ is a bounded convex open subset of $\mathbf{R}^{d}$ and if $v(x)$ is a nonnegative eigenfunction for the Laplace equation: $\Delta v(x)=\lambda v(x), x \in \Omega$, with boundary conditions $\lim _{x \rightarrow a} v(x)=0, a \in \partial \Omega$, then $v(x)$ is a logconcave function.

The basic tool for our study comes from Prékopa, it says that the convolution of two logconcave kernels is a logconcave kernel.

TheOREM 1 (Prékopa [4]). Let be $f(x, y)$ and $g(y, z)$ two logconcave functions, the first defined on $\mathbf{R}^{p} \times \mathbf{R}^{q}$, the second defined on $\mathbf{R}^{q} \times \mathbf{R}^{r}$, then the function $h(x, z)=\int_{\mathbf{R}^{q}} f(x, y) g(y, z) d y$ is logconcave over the set where it is finite.

Received by the editors July 17, 1978.

AMS (MOS) subject classifications (1970). Primary 35K05; Secondary 35K15.

() 1979 American Mathematical Society 0002-9939/79/0000-0216/\$02.00 
If Prékopa's proof is a little complicated, Brascamp and Lieb [1] found a simpler proof which uses the Brunn-Minkowsky-Lusternik inequality.

2. Logconcavity of the kernel of the heat equation. If $\Omega$ is an open subset of $\mathbf{R}^{d}$, one way to solve the heat equation on the cylinder $\Omega \times(0, \infty)$ is to introduce a kernel $p(x, y ; t)=p^{\Omega}(x, y ; t)$. If $\Delta_{x}$ is the Laplace operator with respect to $x$-variable, one looks for a function $p$ such that

$$
\begin{gathered}
\frac{\partial p}{\partial t}(x, y ; t)=\frac{1}{2} \Delta_{x} p(x, y ; t), \quad x \in \Omega, y \in \Omega, t>0, \\
\lim _{x \rightarrow a} p(x, y ; t)=0, \quad a \in \partial \Omega, y \in \Omega, t>0, \\
\lim _{t \downarrow 0} \int_{\Omega} p(x, y ; t) u_{0}(y) d y=u_{0}(x), \quad x \in \Omega .
\end{gathered}
$$

The last equation should hold for any bounded continuous function $u_{0}$ on $\Omega$. The previous equation should hold for any regular boundary point $a$ of $\partial \Omega$.

The construction of such a kernel is well described by Itô and McKean [2, pp. 238-239]. We recall their explanations. We denote by $\|y\|$, the Euclidean norm in $\mathbf{R}^{d}$ of a vector $y$ of $\mathbf{R}^{d}$. If

$$
\begin{aligned}
f_{n}\left(x_{0}, x_{1}, \ldots, x_{n} ; t\right) & \\
=(2 \pi t / n)^{-d / 2} \exp \left\{\left(\left\|x_{1}-x_{0}\right\|^{2}+\left\|x_{2}-x_{1}\right\|^{2}\right.\right. & +\cdots \\
& \left.\left.+\left\|x_{n}-x_{n-1}\right\|^{2}\right) n /(2 t)\right\}
\end{aligned}
$$

where $t>0, x_{i} \in \mathbf{R}^{d}, 0 \leqslant i \leqslant n$, one defines $p_{n}\left(x_{0}, x_{n} ; t\right)$ as the integral in $\mathbf{R}^{d(n-1)}$ of the function $f_{n}$ over the set $\left\{\left(x_{1}, x_{2}, \ldots, x_{n-1}\right): x_{i} \in \Omega, 1<i<n\right.$ $-1\}$. It is convenient to restrict $n$ to powers of 2 . It is easy to check that $p_{2^{k}}(x, y ; t), k=0,1,2, \ldots$, is a decreasing sequence of functions. The limit $p(x, y ; t)=\lim p_{2^{k}}(x, y ; t)$ is the kernel of the heat equation. Something more can be said about this kernel when $\Omega$ is a convex open subset of $\mathbf{R}^{d}$.

THEOREM 2. If $\Omega$ is a convex open subset of $\mathbf{R}^{d}$, the kernel of the heat equation $p(x, y ; t)$ is logconcave with respect to $x$ and $y$.

Proof. If we define the function

$$
g_{n}\left(x_{0}, x_{1}, \ldots, x_{n} ; t\right)= \begin{cases}f_{n}\left(x_{0}, x_{1}, \ldots, x_{n} ; t\right) & \text { if } x_{i} \in \Omega, 0 \leqslant i \leqslant n, \\ 0 & \text { if }(\exists i) x_{i} \notin \Omega ;\end{cases}
$$

$g_{n}\left(x_{0}, x_{1}, \ldots, x_{n} ; t\right)$ is a logconcave function in the variables $x_{0}, x_{1}, \ldots, x_{n}$. By Prékopa's theorem, integration with respect to $x_{1}, x_{2}, \ldots, x_{n-1}$ will leave this property for the result of integration, $p_{n}\left(x_{0}, x_{n} ; t\right), p(x, y ; t)$ as a limit of logconcave functions will still be logconcave.

3. Logconcavity and the cooling of a convex body. We say that a measure $\mu$ on $\mathbf{R}^{d}$ is logconcave if the support of the measure is a convex subset $K$ of $\mathbf{R}^{d}$ and if there is a logconcave function $f(x)$ defined on $K$ such that $d \mu(x)=$ $f(x) d_{H}(x)$ where $H$ is the linear manifold generated by $K$ and $d_{H}(x)$ is the Lebesgue measure on $H$. 
THEOREM 3. If $\Omega$ is a convex open subset of $\mathbf{R}^{d}$, and if $\mu_{0}$ the initial distribution of temperature of $\Omega$ is a logconcave measure carried by $\Omega$, then the distribution of temperature $u(x, t)$ at time $t$ is a logconcave function of $x$ :

$$
u(x, t)=\int_{\Omega} p(x, y ; t) d \mu_{0}(y) .
$$

Proof. Theorems 1 and 2 give the proof.

4. Helmholtz's equation and logconcavity. We now restrict our attention to a bounded open convex subset $\Omega$ of $\mathbf{R}^{d}$. Helmholtz's equation is $\Delta v=\lambda v$ where $v \in C^{2}(\Omega), \lambda \in \mathbf{R}$. It is known that $H_{\lambda}=\left\{v \in C^{2}(\Omega) \cap C(\bar{\Omega}): \Delta v=\lambda y\right.$, $(\forall x \in \partial \Omega) v(x)=0\}$ is a finite dimensional vector space and that $L^{2}(\Omega)$ is the orthogonal sum of all these $H_{\lambda}$. Moreover $H_{\lambda}=\{0\}$ if $\lambda \geqslant 0$ and $\{\lambda$ : $\left.H_{\lambda} \neq\{0\}\right\}$ is a countable set with no accumulation point in any finite interval. Let us denote by $\lambda_{1}, \lambda_{2}, \ldots$ the decreasing sequence of these $\lambda$ such that $H_{\lambda} \neq 0$. If $r_{n}$ is the dimension $H_{\lambda_{n}}$ and if one chooses an orthonormal basis $v_{n, 1}, v_{n, 2}, \ldots, v_{n, r_{n}}$ of $H_{\lambda_{n}}$, the family of functions $\left\{v_{n, i}: 1<i<r_{n}\right.$, $n=1,2, \ldots\}$ is an orthonormal basis of $L^{2}(\Omega)$. The connection between this basis and the kernel of the heat equation is the spectral representation of the kernel

$$
p(x, y ; t)=\sum_{n=1}^{\infty} \sum_{i=1}^{r_{n}} e^{\lambda_{n} t} v_{n, i}(x) v_{n, i}(y) .
$$

These results can be found in Kac [3] for example.

Up to this point, the convexity of $\Omega$ is not needed. When $\Omega$ is convex (or more generally if $\Omega$ is connected), something more is known about the spectral value $\lambda_{1}: r_{1}=1$ and $v_{1}(x)=v_{1,1}(x)$ does not vanish on $\Omega$. One can always assume that $v_{1}(x) \geqslant 0$; if this is not the case, interchange $v_{1}$ with $-v_{1}$. This unique function $v_{1}$ will be called the dominant eigenfunction of Laplace operator.

THEOREM 4. If $\Omega$ is a bounded convex open subset of $\mathbf{R}^{d}$, the dominant (positive) eigenfunction of Laplace operator is logconcave.

Proof. To prove that $v_{1}$ is logconcave, it suffices to prove that $p(x, y ; t) e^{-\lambda_{1} t}$ converges to $v_{1}(x) v_{1}(y)$ as $t$ tends to $\infty$, since the heat kernel is logconcave. By using the spectral representation of $p(x, y ; t)$, we get

$$
p(x, y ; t) e^{-\lambda_{1} t}-v_{1}(x) v_{1}(y)=\sum_{n=2}^{\infty} e^{\lambda_{n} t-\lambda_{1} t}\left(\sum_{i=1}^{r_{n}} v_{n, i}(x) v_{n, i}(y)\right) .
$$

By the Cauchy-Schwarz inequality,

$$
\begin{aligned}
& \left|p(x, y ; t) e^{-\lambda_{1} t}-v_{1}(x) v_{1}(y)\right| \\
& \leqslant\left(\sum_{n=2}^{\infty} \sum_{i=1}^{r_{n}} e^{\lambda_{n} t-\lambda_{1} t} v_{n, i}^{2}(x)\right)^{1 / 2}\left(\sum_{n=2}^{\infty} \sum_{i=1}^{r_{n}} e^{\lambda_{n} t-\lambda_{1} t} v_{n, i}^{2}(y)\right)^{1 / 2} .
\end{aligned}
$$

In order to get bounds on each of these square roots, we use again the 
spectral representation with $y=x$ and $t=1$ :

$$
\sum_{n=1}^{\infty} \sum_{i=1}^{r_{n}} e^{\lambda_{n}} v_{n, i}^{2}(x)=p(x, x ; 1)<(2 \pi)^{-d / 2} .
$$

So, if $M(t)=\sup _{n>2} e^{\lambda_{n} t-\lambda_{1} t-\lambda_{n}}$, then

$$
\begin{aligned}
& \left|p(x, y ; t) e^{-\lambda_{1} t}-v_{1}(x) v_{1}(y)\right| \\
& \quad \leqslant M(t)\left(\sum_{n=2}^{\infty} \sum_{i=1}^{r_{n}} e^{\lambda_{n}} v_{n, i}^{2}(x)\right)^{1 / 2}\left(\sum_{n=2}^{\infty} \sum_{i=1}^{r_{n}} e^{\lambda_{n}} v_{n, i}^{2}(y)\right)^{1 / 2} \\
& \leqslant M(t)(2 \pi)^{-d / 2} .
\end{aligned}
$$

Since $\lim _{t \rightarrow \infty} M(t)=0$, the proof is completed.

\section{REFERENCES}

1. H. J. Brascamp and E. H. Lieb, Some inequalities for Goussian measures and the long-range order of the one-dimensional plasma, Functional Integration and its Applications, Clarendon Press, Oxford, 1975.

2. K. Itô and H. P. McKean, Jr., Diffusion processes and their sample paths, Springer-Verlag, New York, 1965.

3. M. Kac, On some connections between probability theory and differential and integral equations, Proc. Second Berkeley Symposium on Math. Statist. and Probability, Univ. of California Press, 1951, 189-215.

4. A. Prékopa, On logarithmic concave measures and functions, Acta Sci. Math. 34 (1972), 336-343.

Département de Mathématiques, École Polytechnique, C.P. 6079, Succ. “A”, Montréal, QUÉbec, H3C 3A7, CANADA

département de Mathímatiques et de Statistiques, Universtré de Montréal, C.P. 6128, Succ. "A", Montréal, Qú́bec, H3C 3J7, CANADA 